\title{
Study on Clinical Value of Laparoscopic Surgery on Gynecology
}

\author{
Lili Xuan ${ }^{1}$, Xiaojun Liu*,1 \\ ${ }^{1}$ China-Japan Union hospital of Jilin University, Changchun, Jilin, 130033 \\ *corresponding author
}

Keywords: clinical value; Laparoscopic Surgery; Gynecology

\begin{abstract}
To explore the clinical application of laparoscopic surgery in gynecology, 229 cases underwent laparoscopic gynecological fallopian tube surgery, ovarian tumor surgery, uterus (including myomectomy and hysterectomy), and 50 cases of laparoscopic and open surgery with the same surgical indications at the same time. The hemorrhage, postoperative analgesic drug use rate, postoperative infusion day, hospitalization day and postoperative fever rate were compared. Most laparoscopic surgery for benign gynecologic diseases was successful. Compared with laparotomy, the laparoscopic group had less intraoperative blood loss, less postoperative medication, less pain, shorter hospital stay, and lower postoperative fever rate. Laparoscopic surgery is minimally invasive, quick to recover, and has few postoperative complications, which can be used in most gynecological benign diseases.
\end{abstract}

\section{Introduction}

From January 2010 to December 2012, 160 surgical patients admitted to the Department of Gynecology of Yongcheng People's Hospital were selected, aged 23 to 48 years, with an average age of (35. $3 \pm 5.6$ ) years. Among them, there were 9 cases of hysterectomy, 53 cases of uterine fibroids, 48 cases of ovarian cysts, 34 cases of fallopian tube adhesion, 34 cases of hydrops, 6 cases of cervical lesions, and 10 cases of pelvic inflammatory disease. According to different surgical methods, 160 surgical patients were randomly divided into the observation group and the control group. The two groups were statistically processed in terms of age, disease type, and physical condition, and the differences were not statistically significant and comparable.

After the patient was examined, an epidural anesthesia or subarachnoid block anesthesia combined with an epidural anesthesia was performed. The use of scalpels, hemostats, tissue scissors, uterine scissors, wire scissors, and abdominal hooks were routinely performed. Abdominal surgical instruments are used to perform a laparotomy on the patient's lower abdomen with transverse or longitudinal incisions. Postoperative intravenous infusion of conventional antibiotics to prevent infection and take appropriate nursing measures. After the patient's examination, tracheal intubation, intravenous combined with general anesthesia, laparoscopy and the necessary light source, imaging system, the patient cut the skin $1 \mathrm{~cm}$ from the lower edge of the patient's umbilical cord, insert the pneumoperitoneum needle from the incision at $45^{\circ}$, successful puncture After the needle is left in the abdominal cavity, the $\mathrm{CO} 2$ inflator is connected so that the air intake speed is controlled within $1 \mathrm{~L} / \mathrm{min}$. The total amount is 2 to 3L, and the intra-abdominal pressure does not exceed 2. $13 \mathrm{kPa}$ $(1 \mathrm{mmHg}=0.133 \mathrm{kPa})$. Then, raise the abdominal wall of the patient's umbilicus and slowly insert the trocar obliquely and slowly into the abdominal cavity. When entering the abdominal cavity, there is a sense of breakthrough. Pull out the sleeve core and insert the laparoscope after hearing the sound of gas in the abdominal cavity. Turn on the light source. Adjust the position of the patient into a head with a low hip height of $15^{\circ}$, and continue to inflate slowly; the doctor observes the laparoscope after the examination, remove the laparoscope after the examination, remove the gas from the patient's abdominal cavity, remove the cannula, and sew the abdominal incision [1]. Postoperative intravenous infusion of conventional antibiotics to prevent infection and take appropriate nursing measures.

By referring to the data of the cases, the differences in blood loss, average length of stay, and 
postoperative recovery time between the two groups were compared; follow-up visits were conducted through outpatient review, and the postoperative delayed onset was compared between the two groups. The occurrence of complications such as sexual bleeding, internal organ damage, shoulder pain, etc. Adopt SPSS13. 0 statistical software package for statistical analysis, measurement data comparison using $\mathrm{x} 2$ test, measurement data with ( $\mathrm{x}$ 坬 $\pm \mathrm{s}$ ) said, between the two groups using a group $\mathrm{t}$ test, $\mathrm{P}<0.05$ was statistically significant.

\section{Results}

The mean intraoperative blood loss, length of hospital stay, and postoperative recovery time were significantly lower in the observation group than in the control group $(\mathrm{P}<0.05)$, which was statistically significant. The incidence of complications in the two groups was significantly lower in the observation group than in the control group $(\mathrm{P}<0.05)$, which was statistically significant.

For gynecological diseases, adopting effective treatment methods can not only completely eradicate patient's pain, but also be the key to effectively reduce the incidence of complications. The results of this study showed that the intraoperative blood loss, average length of stay, and postoperative recovery time were significantly lower in the observation group than in the control group, and the incidence of complications was lower than in the control group. Compared with traditional laparotomy, laparoscopic surgery for gynecological diseases mainly has the following advantages: First, the incision of laparoscopic surgery is small, which can effectively reduce the amount of blood loss during surgery, and the scar is small, which can satisfy the psychological beauty of modern women. Demand; Secondly, due to the maturation of laparoscopic techniques and the improvement of the professional operation level of doctors, the treatment can be completed within a short period of time, effectively reducing the number of days of hospitalization for patients and reducing the economic burden on patients; again, laparoscopic surgery is performed in a closed abdominal cavity. Conducted, less interference by external factors, minimize damage to the surrounding tissue, faster recovery after surgery; Finally, laparoscope can be checked from different angles and directions without affecting the abdominal organs, to achieve intuitive The effect of the examination is to effectively reduce the incidence of postoperative complications. However, laparoscopic surgery as an emerging method for the treatment of gynecological diseases, due to a variety of factors in the use of the process, will still cause patients with postoperative complications such as delayed bleeding, internal organs damage, shoulder pain, etc. The main reasons are the defects of the equipment itself, improper surgical selection, and insufficiency of doctors' operation techniques.

\section{Laparoscopic Application Value}

Laparoscopic diagnosis and treatment of infertility: Laparoscopic techniques were first applied to the diagnosis of infertility. With the development of laparoscopic techniques and devices, more and more infertility treatments have been used. The cause of infertility can be clearly defined and pelvic disease can be treated without having to open the abdomen [1]. 38 cases of infertility patients treated with this data underwent operations such as adhesion loosening, ovarian hole punching, fallopian tube paranasal endostomy, fallopian tube drainage, electrocoagulation and different surgery, and one operation to remove several causes and avoid laparotomy. The pain has achieved satisfactory results. Laparoscopic tubal anastomosis has been successfully reported [2], which reflects the new progress of laparoscopy in the treatment of infertility.

Laparoscopy in the diagnosis and treatment of ectopic pregnancy value: Application of laparoscopic early diagnosis and treatment of ectopic pregnancy can reduce the incidence of ectopic pregnancy rupture and mortality ratio, while improving the fertility of the fallopian tube is of great significance [3]. Twenty of the 38 patients underwent surgical resection of the fallopian tubes. No fertility request or postembryonic hemorrhage was performed with fallopian tube resection. There was 1 case of uterine horn pregnancy and MTX 20 was injected intramuscularly. Mg was successful. 1 case of menopause for more than 50 days, uterine cavity without scraping gestational sac but high 
blood hCG and B-ultrasonography found no abnormalities, laparoscopic tubal diameter of only 1 $\mathrm{cm}$ in the mass, giving local injection, postoperative blood h The CG quickly dropped to normal. Laparoscopic surgery with early diagnosis and treatment as a whole can not only look directly at the pelvic cavity, but also can promptly select the appropriate surgical method to provide treatment. It is the best choice for ectopic pregnancy, and it is timely for patients with high suspicion and difficult diagnosis. Laparoscopic surgery, conditional hospitals should consider laparoscopic surgery as the first choice for early treatment of ectopic pregnancy.

Laparoscopy in the application of ovarian tumors: Ovarian tumor resection or removal is a common operation in gynecology, in the past smaller cysts were followed up, until the cyst grew to $5 \mathrm{~cm}$ or complications when open surgery [4] . After laparoscopic surgery, some small ovarian tumors were promptly treated. In this report, 1 patient had a teratoma with a diameter of $3 \mathrm{~cm}$ in the left ovary before B-ultrasonography. After conventional excision of the contralateral ovary There was a small teratoma with a diameter of only $0.5 \mathrm{~cm}$. No preoperative B ultrasound was detected and it was removed during the procedure. The controversy of laparoscopic treatment of ovarian tumors is that the nature of the tumor cannot be determined before surgery. If it is a malignant tumor, the operation may be incomplete or may result in disseminated or recurrence of the tumor after microsurgery [5]. Therefore, preoperative evaluation of the nature and quality of the tumor Confirming the diagnosis is the key to selecting laparoscopic surgery. It has been reported that the accuracy of B-predicted benign ovarian cysts can reach 96\%. Teratoma and endometriotic cysts are predominant in benign ovarian tumors. In the present study, the two groups accounted for $30 \%$ and $40 \%$ of ovarian masses respectively. Teratoma can cause mass rupture when removed under the microscope. Lipid-like substances can easily flow into the abdominal cavity. However, as long as the pelvic cavity is flushed, there are no other complications. There are 5 cases of rupture in this data, no obvious complications appear, once again confirmed this point. Endometriosis cysts if the cyst is much larger and pelvic organs adhesion and easy to rupture in the separation, you can first puncture cysts, absorb the cyst fluid, and then remove the cyst wall trimmed ovaries or attachment removal. Although the maximum diameter of excised cysts in this data is $15 \mathrm{~cm}$, compared with open surgery, indications for ovarian tumors are still limited. For patients with severe pelvic adhesions or suspicious malignancy, they should not be forced to operate under the microscope. There were 3 cases of severe adhesion to the laparotomy laparotomy, accounting for 1 . 31\%. For those who have a history of surgery, as long as the puncture into the mirror is successful, the vision can be seen under the microscope, and the loose adhesion can also be separated. There are 25 patients with history of lower abdominal surgery in this data. All of the patients underwent successful microscopic surgery, including 1 case of uterus. After resection of the pelvic recurrence of patients with mass, I have concerns about re-opening, laparoscopic puncture success, careful careful separation of the abdominal cavity adhesions, and ultimately the pelvic chronic inflammation of the hydrotubation of the fallopian tube. Already, under the microscope, pelvic lymphadenectomy and extensive hysterectomy have been performed. With the development of laparoscopic techniques, it is expected that more extensive surgery can be performed under the microscope.

The application of laparoscopy in uterine surgery: The removal of uterine fibroids is not a simple operation in laparoscopic surgery. It is easy to remove fibroids in the subserous and broad ligament and it is easy to stop bleeding, but for the intermuscular myoma , must select the case of convex to the serosal surface, under the microscope has a good suture technique can be operated, the removal of the great fibroids is best carried out under the conditions with a cylindrical rotary cutter, 15 cases in this group of data All the patients with myomectomy had good results. The largest fibroid size was $10 \mathrm{~cm}$ in diameter, and the operation was successfully completed with a rotary cutter. L AV H has been reported in the country. In this document, 15 cases of hysterectomy were performed with $\mathrm{L}$ AV $\mathrm{H}$ method. The largest uterus was excised for 12 weeks and the operation was successful. Currently, laparoscopic intrafascial hysterectomy (CASH) is gradually being promoted in China. Compared with $\mathrm{L}$ AV $\mathrm{H}, \mathrm{CASH}$ has the advantages of common hysterectomy and subtotal hysterectomy, which can prevent the cervix. The occurrence of epithelial stump cancer in the 
external osseous transition maintains the integrity of the pelvic floor tissue, so that the patient still enjoys high quality of life after hysterectomy. At present, we are carrying out this surgical procedure for hysterectomy.

\section{Conclusion}

Laparoscopic surgery such as laparoscopic surgery has advantages of less trauma, quicker recovery, less pain, and higher cure rate. Laparoscopic surgery, as a representative of minimally invasive surgery, has been widely used in the surgical field and involves many diseases and operations, welcomed by patients, and with the continuous advancement of science and technology, surgical instruments to improve innovation, laparoscopic display space will be more and more large. On the day of surgery, patients who are not completely awake during general anesthesia should go to the supine position with their heads to one side, fasting, and water to prevent accidents. Due to the influence of the drug, some patients will have different degrees of nausea and vomiting after operation, and food intake may be postponed appropriately. No nausea and vomiting can be eaten after 6 hours. The postoperative rest time varies from person to person, and one week after surgery can participate in general activities but it is not advisable to do heavy manual labor. The treatment of gynecological diseases in our hospital is based on specific conditions using laparoscopic surgery for treatment, significantly improve the treatment effect, the clinical effect is accurate, safe and effective, it is worthy of widespread clinical application.

\section{References}

[1] Watson. Laparoscopic surgery indications and clinical application. Chinese Journal of Practical Gynecology and Obstetrics, 2006. 22(1): 15- 18

[2] Zuo Xulei, Chief Editor, Practical Gynecologic Laparoscopy. Shenyang: Liaoning Science and Technology Press, 2004. 93- 94, 163-165

[3] Zhou Yingfang. Current status of clinical diagnosis and treatment of endometriosis[J]. Chinese Journal of Obstetrics and Gynecology, 2005, 40(1):67- 70.

[4] Leng Jinhua, Lang Jinghe. Diagnosis and treatment of laparoscopic complications[J]. Chinese Journal of Modern Operative Surgery, 2001, 5(1):69-72.

[5] Zhang Caiyu. Gynecological laparoscopic surgery complications of clinical analysis [J]. Maternal and Child Health of China, 2011, (26): 360 - 361. 